\title{
Bidirectional Best-fit Heuristic for Orthogonal Rectangular Strip Packing
}

\author{
Önder Barış Aşık \\ Yeditepe University \\ Department of Computer Engineering \\ İnönü Cad. Kayışdağı Mah. \\ 34755 Kadıköy/İstanbul Turkey \\ Tel: +90 2165780423 \\ Fax: +90 2165780400 \\ oasik@cse.yeditepe.edu.tr
}

Ender Özcan (corresponding author)*

ASAP Research Group

School of Computer Science

University of Nottingham

Jubilee Campus, Wollaton Road, Nottingham NG8 1BB UK

Tel: +44 2165780423

Fax: +44 2165780400

exo@cs.nott.ac.uk 


\begin{abstract}
In a non-guillotinable rectangular strip packing problem (RF-SPP), the best orthogonal placement of given rectangular pieces on a strip of stock sheet having fixed width and infinite height are searched. The aim is to minimize the height of the strip while including all the pieces in appropriate orientations. In this study, a novel bidirectional best-fit heuristic (BBF) is introduced for solving RF-SPPs. The proposed heuristic as a new feature considers the gaps in both horizontal and vertical directions during the placement process. The performance of BBF is compared to some previous approaches, including one of the best heuristics from the literature. BBF achieves better results than the existing heuristics and delivers a better or matching performance as compared to the most of the previously proposed meta-heuristics for solving RF-SPPs.
\end{abstract}

Keywords Heuristic, sequencing, strip packing, non-guillotine cutting

Abbreviations

$\mathrm{BBF}$,

bidirectional best-fit heuristic;

SPP,

rectangular strip packing problem;

RF, non-guillotinable, variable orientation subtype;

RF-SPP non-guillotinable, variable orientation (orthogonal) SPP

GA, genetic algorithm;

SA, simulated annealing;

GRASP, greedy randomized adaptive search procedure

BF, best fit heuristic;

BL, bottom left heuristic;

iBL, improved bottom left heuristic;

BLF, bottom left fill heuristic; 


\section{Introduction}

Cutting and packing a given stock material are crucial processes in manufacturing and producing. Paper, plastic, wood, glass, steel and leather are some of the most commonly used stock materials in the industries. Different types of cutting and packing problems arise due to the nature of the stock material, such as different constraints and objectives. For example, regular shapes are to be cut in the paper industry while irregular shapes are to be cut in the leather and textile industries. Surveys on the cutting and packing problems can be found in Hinxman (1980), Sarin (1983), Dyckoff (1990), Hässler and Sweeney (1991), Dowsland and Dowsland (1992), Hopper and Turton (2001b) and Lodi, Martello and Monaci (2002).

\subsection{Problem definition}

Solving a rectangular strip packing problem (SPP) requires a search for the best placement of a set of rectangles with given widths and heights in a suitable orientation on a strip of rectangular stock sheet with a fixed width and an infinite height. The ultimate objective is minimizing the wastage while maximising the material utilization. Based on the recent categorization of the cutting and packing problems provided by Waescher, Haussner and Schumann (2007), SPP is identified as two-dimensional, open dimension problem. If the rotation of rectangles by 90-degrees is allowed and guillotine cutting (edge-to-edge) is not required as constraints during the placement process, this type of problems is referred to as RF subtype (Lodi, Martello, Vigo 1999 and Bortfeld 2006). In this paper, a new heuristic is presented for solving orthogonal rectangular strip packing problem of RF subtype. Orthogonal placement requires the sides of the rectangles to be parallel to the edges of the strip. The ultimate objective is to minimise the height of the strip on which all rectangles will be oriented without any overlaps.

For defining the problem more formally, the following notation is used:

- $S$ is the stock sheet having a width of $W$ and its bottom left and right corner at locations $(0,0)$ and $(W, 0)$, respectively. 
- $R$ is the set of $n$ rectangles to be placed; $\mathrm{R}=\left\{r_{1}, \ldots, r_{i}, \ldots, r_{n}\right\}$, where $r_{i}$ is the $i$ th rectangle with a width and height of $\left(w_{i}, h_{i}\right) ; w_{i}, h_{i} \in Z^{+}$and $\min \left\{w_{i}, h_{i}\right\} \leq W$, for $1 \leq i \leq n$.

- 90-degrees of rotations are allowed during the placement. Let $\left(w_{i}^{\prime}, h_{i}^{\prime}\right)$ be the width and height of the $i$ th rectangle after the placement. If the $i$ th rectangle is not rotated, then $w_{i}^{\prime}=w_{i}$ and $h_{i}^{\prime}=h_{i}$. If it is rotated by 90degrees, then $w_{i}^{\prime}=h_{i}$ and $h_{i}^{\prime}=w_{i}$.

- region $\left(r_{i}\right)$ represents the region covered by the $i$ th rectangle and its bottom left corner at location $\left(x_{i}, y_{i}\right)$ within the coordinate system of $S$ after the placement, where $y_{i} \geq 0$ and $0 \leq x_{i} \leq W$.

In an RF subtype orthogonal rectangular strip packing problem (RF-SPP), the goal is to place all rectangles in $R$ onto $S$ in such a way that

$$
\begin{aligned}
& \min \left\{\max _{1 \leq i \leq n}\left\{y_{i}+h_{i}^{\prime}\right\}\right\} \\
& \text { subject to } \\
& \qquad \operatorname{region}\left(r_{i}\right) \cap \operatorname{region}\left(r_{j}\right)=\varnothing, \text { for } 1 \leq i, j \leq n \text { and } i \neq j .
\end{aligned}
$$

\subsection{Previous work}

There are exact and inexact approaches in literature for RF-SPP. Beasley (1985a, $1985 b)$ investigated tree-search for the non-guillotine cutting case and dynamic programming approaches for the guillotine cutting case, respectively. Martello, Monaci and Vigo (2003) proposed an exact approach based on a branch and bound algorithm, while Lesh et al. (2004) combined a pruning method with a branch and bound scheme for solving a subset of small RF-SPPs. Kenmochi et al. (2007) also used a branch and bound approach, where branching is based on two placement schemes and bounding operations are based on dynamic and linear programming. As the number of rectangles to pack grows, exact approaches become impractical. Due to the NP-hard nature of SPP (Garey and Johnson, 1979), researchers have been investigating meta-heuristics, hyper-heuristics and heuristics as inexact approaches to obtain near optimal solutions. Dagli and Poshyanonda (1997) proposed two different hybrid approaches based on artificial neural networks for solving RF-SPPs. Simulated annealing, tabu search and the genetic algorithms are the most commonly used meta-heuristics in previous studies (Jakobs 1996, Liu and Teng 1999, Hopper and Turton 2001a, 2001b, Burke, Kendall and Whitwell 2004, 
2006). Beltran et al. (2004) hybridize greedy randomized adaptive search procedure (GRASP) and variable neighbourhood search to solve different subtypes of strip packing problems. A hyper-heuristic is a high level (meta-)heuristic that explores the search space generated by a set of low level heuristics for choosing, adapting and/or learning them (Ozcan, Bilgin and Korkmaz 2008 and Burke et al. 2003). Garrido and Riff (2007), Terashima-Marin, Moran-Saavedra and Ross (2005), Terashima-Marin, Flores-Alvarez and Ross (2005) evolved sequences of packing heuristics using a genetic algorithm and a classifier system, respectively, that were used as a hyper-heuristic for solving RF-SPPs. Imahori, Yagiura and Ibaraki (2005) investigated multi-start local search and iterated local search approaches using different neighbourhood operators. Their experimental results showed that the iterated local search performed better. Bortfeldt (2006) experimented with a genetic algorithm for solving a variety of SPPs having different constraints. Alvarez-Valdes, Parreno and Tamarit (2008) successfully applied their GRASP approach for solving strip packing problems subject to different constraints.

Zhang, Kang and Deng (2006) described a divide and conquer heuristic (HR) with a worst case running time complexity of $\mathrm{O}\left(n^{3}\right)$. Most commonly known greedy heuristics with polynomial time worst case running times are: the bottomleft (BL), the bottom-left-fill (BLF) and the best-fit (BF) proposed by Baker (1980), Chazelle (1983) and Burke, Kendall and Whitwell (2004), respectively. Both former heuristics are blind placement heuristics, while the latter is a smart one. In BL and BLF, a location is determined for each rectangle from a list provided successively in a predefined order. On the other hand, BF makes informed decisions on which shape to place next. Lesh et al. (2005) proposed a stochastic heuristic (BLD*) that attempts to improve bottom-left-fill approach by perturbing the order of rectangles and moving towards a better sequence of rectangles. In this study, these heuristics will be given focus.

BL heuristic as described by Jakobs (1996), and illustrated in Figure 1 (a), starts at the upper corner of the object, then the piece slides vertically, all the way down, until it hits another piece. It continues sliding to the left (in straight line) as far as possible. A sequence of down and left movements is repeated until the piece reaches a stable position. Liu and Teng (1999) improved the BL heuristic to be used as decoder based on permutation representation in meta-heuristics. Improved 
BL (iBL), instead of moving a piece all the way and straight to the left, keeps sliding it over the borderline of the bottom pieces until it reaches a stable position (Figure 1 (b)). Chazelle (1983) presented a bottom left fill heuristic (BLF) that attempts to utilize the regions left unfilled during the placement process as illustrated in Figure 1(c), requiring a space of $\mathrm{O}(n)$. Being more sophisticated than the previous two heuristics, BLF attempts to fill free spaces between pieces that are already placed, generating better results as compared to BL (Hopper and Turton 2001a).

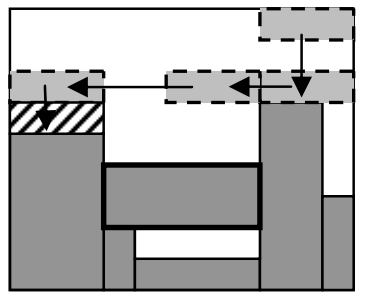

(a)

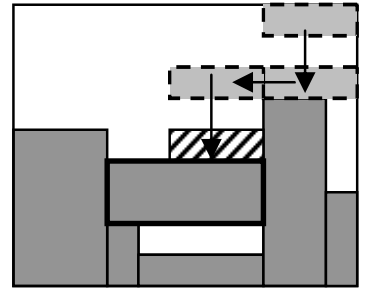

(b)

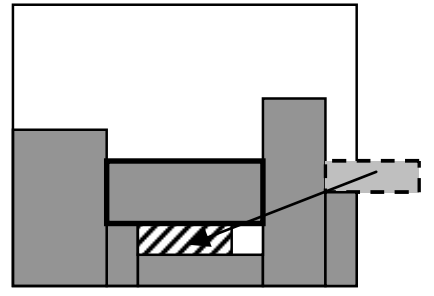

(c)

Fig. 1 Placement of a shape marked with texture after the last placed shape having sides marked with bold line style using (a) bottom left, (b) improved bottom left, and (c) bottom left fill heuristics.

\subsection{Best fit heuristic for RF-SPP}

It has been reported that BF is the best heuristic for solving RF-SPPs (Burke, Kendall and Whitwell 2004). BF consists of three stages: pre-processing, packing and post-processing. During the pre-processing stage, the rectangles are arranged so that the width of each rectangle is larger than its height by 90-degree rotation. Then they are sorted by decreasing width. In case of equality, they are sorted by decreasing height. The width of the lowest niche, referred to as gap is used to make the decision of which rectangle (or rectangle pair) to place. After the lowest gap is identified, BF places the best fitting rectangle into the niche.

Burke, Kendall and Whitwell (2004) shows empirically that the policy used for deciding which part of the gap to place a selected shape generates a performance variance. Hence, a set of horizontal niche placement policies is utilized as it is shown in Figure 2: place at leftmost (LM), place next to tallest neighbour (TN), and place next to shortest neighbour $(\mathrm{SN})$. After the packing stage, the possible towers are eliminated during the post-processing stage. BF is illustrated in Figure 3.

Figure 4 presents a simple RF-SPP instance, labelled as M1 that contains 6 shapes having four $4 \times 1$, one $8 \times 1$ and one $9 \times 1$ rectangles to be packed into a stock 
sheet with a width of 4 . BF fails to obtain the optimal solution for this problem instance. Although the tower elimination in BF is useful in some problem instances, it might become useless for some other cases like M1. BF prefers to place exact fitting rectangles first, hence four $4 \times 1$ are packed into the stock sheet on top of each other, and then assuming the TN policy is used, two tall $(1 \times 9,1 \times 8)$ rectangles are packed at each side of the stock sheet. The result does not improve even if another placement policy is used. In this study, a new heuristic is described that combines different policies to overcome the shortcomings of BF. The approach is tested over a variety of benchmark problems and compared to the previously proposed heuristics and meta-heuristics.

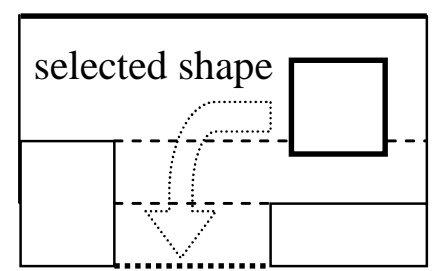

lowest horizontal gap

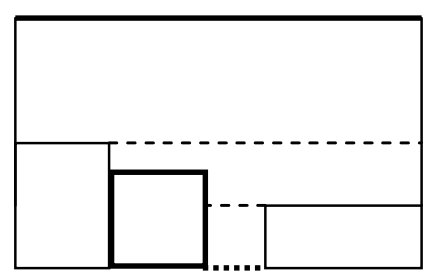

(a)

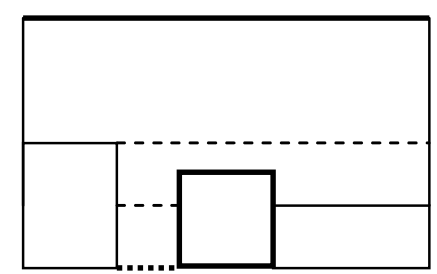

(b)

Fig. 2 Niche placement policies: placement next to (a) tallest (or leftmost) (b) shortest neighbour.

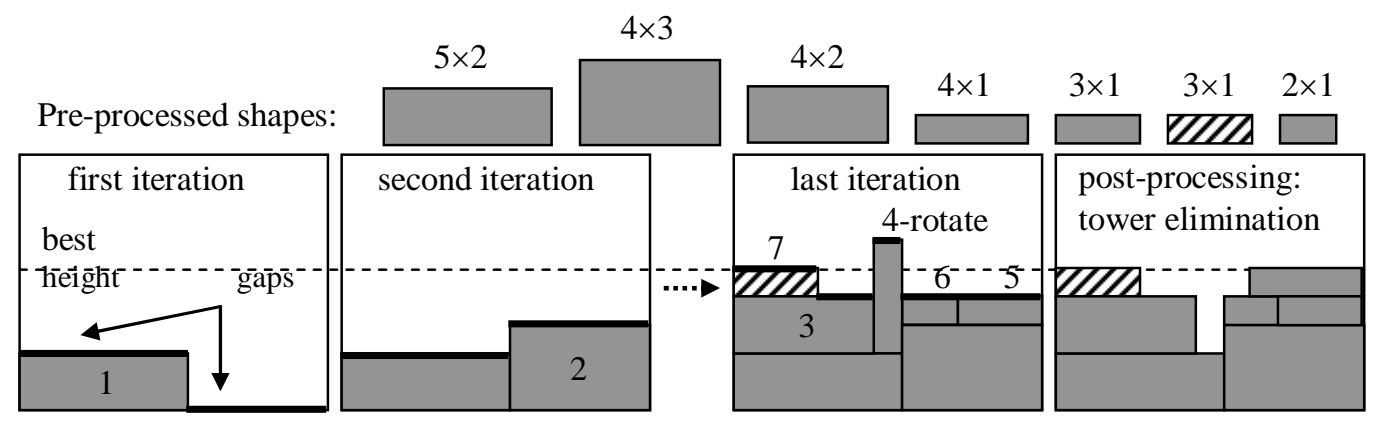

stock sheet width $=9$

Fig. 3 Placement of a set of rectangles using best fit heuristic based on TN policy.

M1: 6 rectangles, $W=4$

\begin{tabular}{cccc}
\hline Width & Height & Width & Height \\
\hline 1 & 4 & 4 & 1 \\
4 & 1 & 1 & 9 \\
1 & 4 & 8 & 1 \\
\hline
\end{tabular}

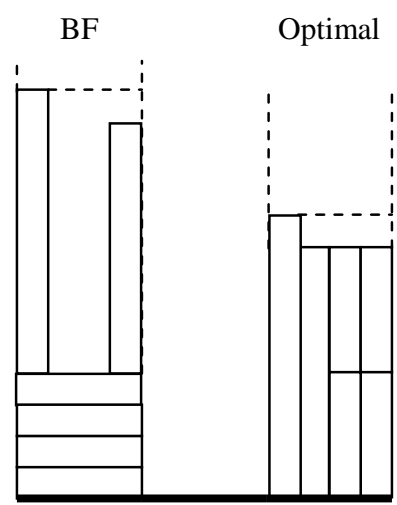

Fig. 4 A simple data (M1) illustrating the shortcoming of best fit heuristic. 


\section{A novel heuristic for RF-SPP}

The aim of the approximation heuristics for solving RF-SPPs is to obtain an optimal skyline (height) after the placement of rectangles. Martello and Vigo (1998) provided better lower bounds for SPP variants. The proposed heuristic makes use of a target height, named as expected best height. The simple lower bound, also known as the continuous bound is preferred as the expected best height. This bound can be computed fast in advance using Equation (2), since the dimensions of a stock sheet and the rectangles to be placed are known before.

$$
\text { expected best height }=\left\lceil\sum_{\forall i, r_{i} \in R} \frac{\text { areaOf }\left(r_{i}\right)}{W}\right\rceil
$$

where $R$ is the set of rectangles, $r_{i}$ is the $i$ th rectangle and $W$ is the width of the stock sheet. The optimal height for an RF-SPP instance can not be lower than this value for discrete width and height. The definition for the expected best height requires a minor adjustment for a better bound. As a simple example, given a set of two rectangles $\{8 \times 2,4 \times 2\}$ and a stock sheet of width 4 , the expected best height computes to 6 . Obviously, $8 \times 2$ rectangle can not be placed into a gap having a maximum width of 4 and the optimal height can not be less than 8 . Therefore, the expected best height should be updated. If the maximum side length among all rectangles, denoted as $\max l=\max \left\{\forall i, r_{i} \in S\right.$ : widthOf $\left(r_{i}\right)$, heightOf $\left.\left(r_{i}\right)\right\}$ is larger than both the expected best height and the width of the stock sheet, then the expected best height is reset to maxl.

The best fit (BF) heuristic aside, bottom-left (BL) and bottom-left fill (BLF) heuristics rely on the sequence of the rectangles provided. BL and BLF heuristics do not require any pre-processing unless the user sorts the rectangles with respect to their decreasing (or increasing) width (or height). However, in the best-fit heuristic a more appropriate rectangle is chosen for placement during the packing stage. This dynamic selection mechanism within the best fit heuristic provides an advantage over the other placement heuristics whose performance might change depending on the input sequence. BF heuristic requires a pre-processing time. The rectangles are arranged by a 90 -degree rotation in such a way that, the width of a rectangle is always larger or equal to its height. Then the rectangles are sorted with respect to their widths in decreasing order. In case of equality, they are sorted with respect to their heights in decreasing order. This same process is also adapted by 
our approach. Our heuristic, named as bidirectional best-fit (BBF) heuristic improves the best-fit approach by using some additional features. Almost all previous heuristics for RF-SPP consider the gaps for placement along a single direction. The proposed greedy heuristic aims to select and place the most appropriate rectangle from a list of unplaced rectangles. During this process, both horizontal and vertical niches on the stock sheet are considered at each step. A preprocessing phase is required by $\mathrm{BBF}$ that is the same as the $\mathrm{BF}$ approach; nevertheless, $\mathrm{BBF}$ requires no post-processing like tower elimination in $\mathrm{BF}$.

\subsection{Maintenance of the niches}

The horizontal gaps and hence the lowest niche(s) are maintained as in BF. An array or a linked list can be used as a data structure to keep track of all gaps in terms of their height (y-coordinate) and length as shown in Figure 5.. Additionally, a single vertical niche is also used by the proposed heuristic. A vertical niche is a single rectangular region formed by the upper-left-most niche between the upper-left-most horizontal gap and the expected best height. The height and width of a vertical niche is illustrated in Figure 5.

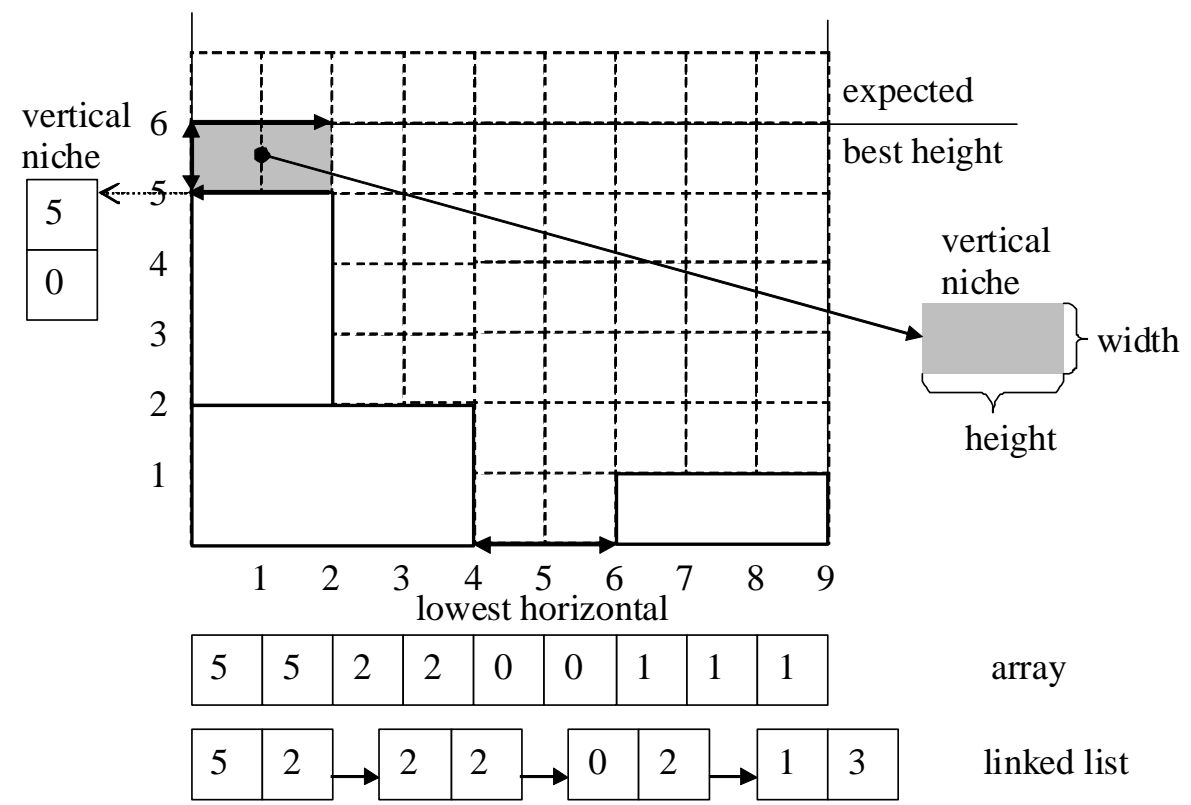

Fig. 5 Data structures that can be utilized for maintaining niches.

\subsection{Bidirectional best-fit heuristic (BBF)}

After pre-processing as described previously, BBF invokes the placement process. At each step, a rectangular region (or niche) is considered for placement and a 
decision is made regarding to which rectangle is to be placed, its orientation and its precise spot within that region based on a set of policies. This decision can be delayed, if the rectangular region (or niche) is too small for placement. Consequently, the placement process consists of three consecutive core stages as illustrated in Figure 6:

1. In the first stage, an exact fit is targeted based on some rectangle selection policies by considering both the lowest horizontal gap and the vertical gap.

2. If the algorithm fails in the first stage, then a best fit is targeted in the second stage.

3. In the third stage, the width of the horizontal gap can be so small that no rectangle fits in. Then the horizontal gap is wasted by raising the height of the niche to the shortest neighbour's level. The search proceeds with the new lowest horizontal gap, formed after this modification as described. The vertical gap remains unchanged even if there is no fitting rectangle.

The major differences between the new heuristic and the previous approaches are that BBF utilizes a search for a bidirectional placement and there is a clear distinction between searching for an exact fitting and best fitting rectangle supported by a set of new policies. The best fit in BBF indicates a fitting rectangle that does not meet the criteria as defined for an exact fit. Performing the placement along either dimension at earliest might affect the result; hence, this ordering is also taken into account. Policies are arranged such that the gaps are filled as much as possible by using the larger objects first and keeping the number of gaps less.

1. BBF_Solve()

2. Input: stock sheet dimensions: $W$, list of $n$ rectangles Output: height

//-- start of pre-processing

3. foreach rectangle in the list

4. if (rectangle width $>$ rectangle $_{\text {height }}$ )

5. rotate the rectangle by $90^{\circ}$ and $\operatorname{swap}\left(\right.$ rectangle $_{\text {width }}$, rectangle height $\left._{\text {. }}\right)$

6. sort the rectangles in the list by non-increasing width and in case of equality sort them by height

$/ /--$ end of pre-processing

7. foreach rectangle selection policy\# 1 for exact fit into the vertical niche in $\{$ enabled, disabled $\}\{$

8. foreach rectangle selection policy\#2 for exact fit into the horizontal niches in \{TRE, NRE\}\{

9. foreach exact-fit ordering policy\#3 in $\{\mathrm{eHV}, \mathrm{eVH}\}\{$

10. foreach horizontal best-fit policy\#4 in $\{\mathrm{BP}, \mathrm{FP}\}\{$

11. foreach vertical best-fit policy\#5 in $\{\mathrm{FH}, \mathrm{WR}, \mathrm{noVB}\}\{$

12. foreach best-fit ordering policy\#6 in $\{b H V, b V H\}\{$

13. foreach placement policy\#7 for a selected rectangle in $\{\mathrm{LM}, \mathrm{TN}, \mathrm{SN}\}\{$

14. initialize gaps, reset list to sorted rectangles, fit_found=false, placed_rectangles $=0$

$15 . \quad$ while (placed_rectangles! $=n)\{$ 


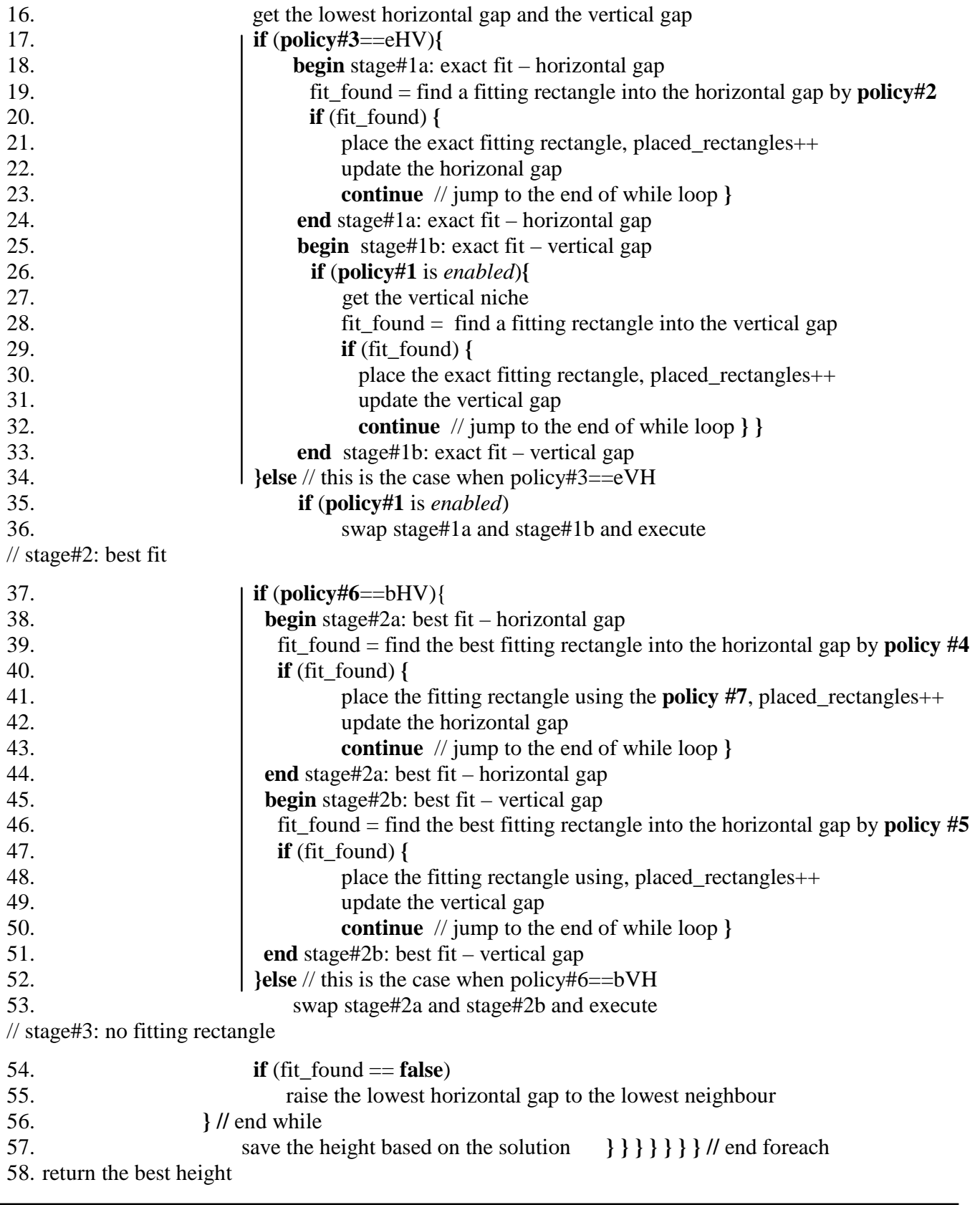

Fig. 6 Pseudocode of the bidirectional best-fit heuristic.

The aim in the first stage is filling a gap entirely. This stage contains two substages: exact fit search for the horizontal gap and exact fit search for the vertical gap. An exact fit ordering policy is administered during this stage, since filling a vertical gap might be advantageous over filling a horizontal gap first, or vice versa $(\mathrm{eVH}, \mathrm{eHV})$. Two different rectangle selection policies are utilized while filling the horizontal gap. The traditional policy in BF selects the first rectangle that fits into the lowest gap from the sorted list of single rectangles. This policy is labelled as TRE. In BBF, an additional policy is utilized, denoted as NRE. In NRE, two different choices are considered consecutively before the first fitting rectangle is 
checked as illustrated in Figure 7. Initially, a rectangle having a height of the tallest neighbour of the lowest horizontal gap is searched, and then the shortest one. This strategy aims to generate a wider horizontal gap after the placement. If both attempts fail, then the fitting rectangle with the tallest height is given higher priority to be placed. The sorted list is searched for a suitable rectangle starting from the first unused one. If there is an exact fitting rectangle, then due to the inclusion of this last attempt, NRE turns out to be the TRE policy. If there is no fitting rectangle, then no other action is taken during this stage.
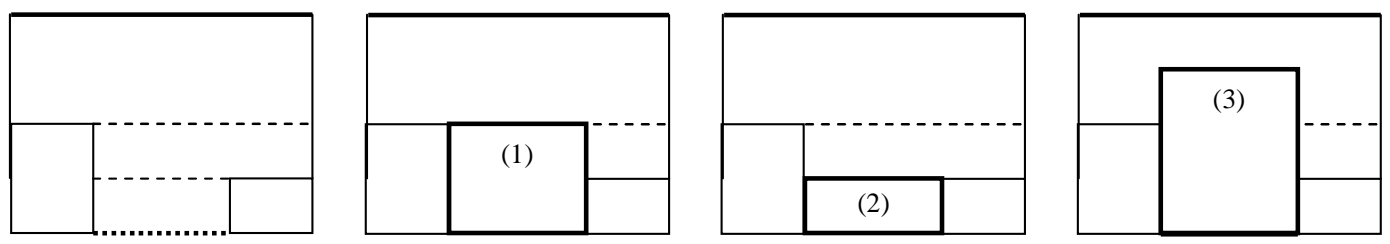

lowest horizontal gap

Fig. 7 NRE rectangle selection policy steps for exact fit into the lowest horizontal niche, consecutively, look for a rectangle: (1) having a height of the tallest neighbour, (2) having a height of the shortest neighbour, (3) and then apply the TRE policy.

Unused large objects generate difficulty towards the end of placement, since it becomes likely that the number of gaps increases in the horizontal direction causing wastage. This is the main reason why a single vertical niche is considered for placement during the first stage as another sub-stage. In this sub-stage, a rectangle is searched in accordance with a size exactly equal to the vertical niche width. If the attempt fails, then a search is performed for a rectangle with a height that is as close as possible to the height of the vertical niche. If such a rectangle is found, then the new vertical niche is constructed as shown in Figure 8. The search for an exact fitting rectangle into the vertical niche can be enabled or disabled as a policy.

In the second stage, the best fitting rectangle is searched for among the unplaced rectangles. This stage also contains two sub-stages: best fit search for the horizontal gap and the vertical gap. Similarly, an ordering policy is used to check both possibilities of invoking the search for the horizontal gap or the vertical gap first (bHV, bVH). The third policy disables vertical best fit, denoted as noVB. Two different policies are used for selecting the best rectangle for placement for the horizontal gap. The first policy denoted as BP is the traditional one utilized by the best fit heuristic; search for a rectangle that minimizes the remaining gap. The second policy denoted as FP places the first fitting rectangle 
from the sorted list considering the rotated versions as well. Hence, possibly, the tallest rectangle gets inserted. The search for the best rectangle that fits into the vertical niche is based on two different rectangle selection policies. The first policy denoted as FH fixes the height of the rectangle to be searched equal to the height of the vertical niche. The rectangle with the largest width from the sorted list is inserted into the niche. The second policy denoted as WR inserts the widest rectangle that fits into the niche ignoring its height. As presented in Figure 6, the proposed heuristic attempts to test all policy combinations summarized in Table 1 and return the best result.

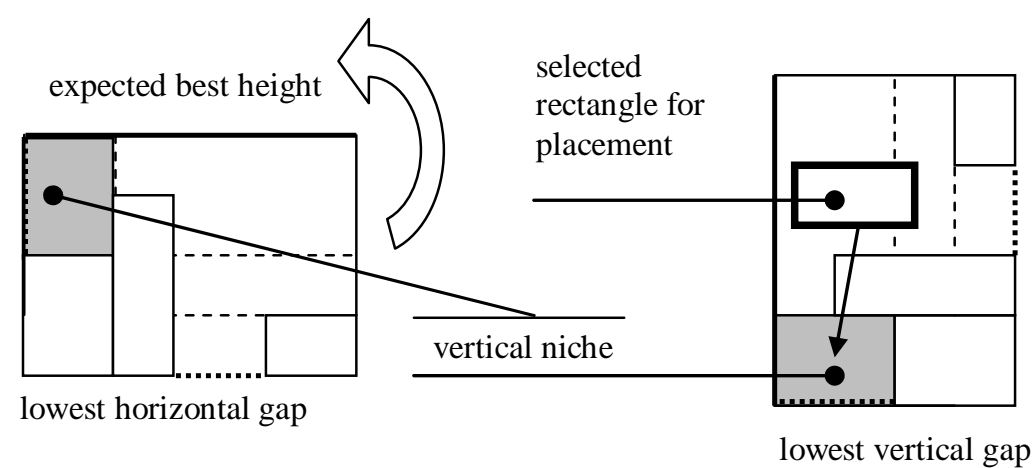

(a)

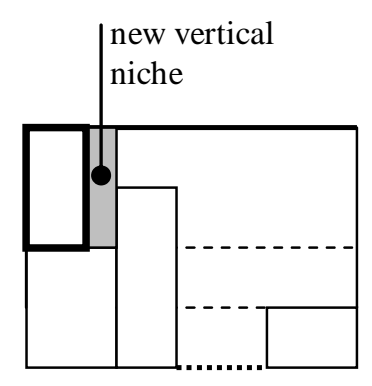

(b)

Fig. 8 (a) Placement of a selected rectangle into the vertical niche, and (b) vertical niche update.

Table 1 Brief explanation of the policies utilized by BBF.

\begin{tabular}{|c|c|c|c|}
\hline Id. & Policy & Value & Brief explanation \\
\hline \multirow[t]{2}{*}{1} & \multirow{2}{*}{$\begin{array}{l}\text { rectangle selection } \\
\text { policy for exact fit into } \\
\text { the vertical niche }\end{array}$} & Enabled & $\begin{array}{l}\text { Placement into the vertical niche is considered } \\
\text { during the exact fit phase }\end{array}$ \\
\hline & & Disabled & $\begin{array}{l}\text { Only placement into horizontal niche is considered } \\
\text { for an exact fit, and policy\#3 is ignored }\end{array}$ \\
\hline \multirow[t]{2}{*}{2} & \multirow{2}{*}{$\begin{array}{l}\text { rectangle selection } \\
\text { policy for exact fit into } \\
\text { the horizontal niche }\end{array}$} & TRE & The first rectangle that fits into the niche is chosen \\
\hline & & NRE & $\begin{array}{l}\text { An exact fit into two different rectangular regions } \\
\text { are considered consecutively, if not possible, the } \\
\text { tallest rectangle that fits into the niche is chosen }\end{array}$ \\
\hline \multirow[t]{2}{*}{3} & \multirow[t]{2}{*}{$\begin{array}{l}\text { Exact fit ordering } \\
\text { policy }\end{array}$} & $\mathrm{eHV}$ & $\begin{array}{l}\text { Possibility of an exact fit into the horizontal niche } \\
\text { is sought first, if it is not possible, then the } \\
\text { possibility of an exact fit into the vertical niche is } \\
\text { considered }\end{array}$ \\
\hline & & $\mathrm{eVH}$ & Opposite of eHV \\
\hline \multirow[t]{2}{*}{4} & \multirow[t]{2}{*}{$\begin{array}{l}\text { Horizontal (gap) best } \\
\text { fit policy }\end{array}$} & $\mathrm{BP}$ & $\begin{array}{l}\text { Choose the rectangle that minimizes the remaining } \\
\text { gap }\end{array}$ \\
\hline & & FP & Choose the first fitting tallest rectangle \\
\hline \multirow[t]{2}{*}{5} & \multirow[t]{2}{*}{$\begin{array}{l}\text { Vertical (gap) best fit } \\
\text { policy }\end{array}$} & FH & $\begin{array}{l}\text { Fix the height, choose the rectangle with this } \\
\text { height and the largest width }\end{array}$ \\
\hline & & WR & $\begin{array}{l}\text { Chooses the widest rectangle that fits into the } \\
\text { vertical niche }\end{array}$ \\
\hline 6 & Best fit ordering & noVB & Best fit into the vertical niche is disabled \\
\hline
\end{tabular}




\begin{tabular}{lll} 
policy & bHV & $\begin{array}{l}\text { Possibility of a best fit into the horizontal niche is } \\
\text { sought first, if it is not possible, then the } \\
\text { possibility of a best fit into the vertical niche is } \\
\text { considered }\end{array}$ \\
Placement policy & LVH & $\begin{array}{l}\text { Opposite of bHV } \\
\text { (horizontal gap) }\end{array}$ \\
& TN & $\begin{array}{l}\text { Next to the left most neighbour } \\
\text { Next to the tallest neighbour }\end{array}$ \\
\hline
\end{tabular}

\subsection{Illustration of how BBF executes}

Given M1 problem instance (Figure 1) containing single rectangles of sizes $1 \times 9$, $8 \times 1$, two from $4 \times 1$ and two from $1 \times 4$ rectangles and a strip of width $4, \mathrm{BBF}$ obtains a solution as follows. Note that the maximum side length is 9. First, an expected best height is computed as 9 using $\max \{\lceil 33 / 4\rceil, 9\}$ (see Section 2). After pre-processing, following sorted list of rectangles is generated $\{9 \times 1,8 \times 1,4 \times 1$ $4 \times 1,4 \times 1,4 \times 1\}$. Figure 9 shows the logical view of some internal data structures required for representing and solving the problem just before testing a policy combination at step 14 in Figure 6. Let's assume that the policy combination represented by ordered 7-tuple <Enabled, TRE, eVH, FP, WR, bHV, TN> will be tested (see Table 7).

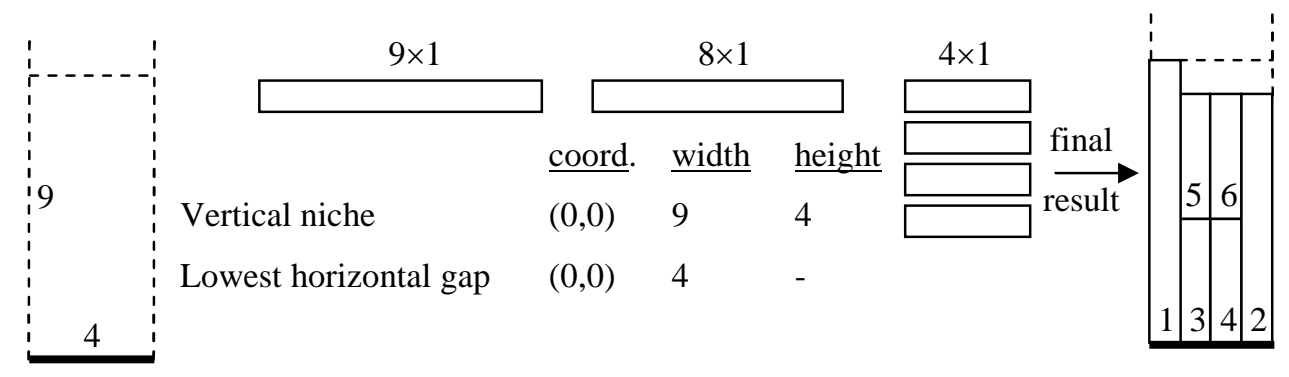

Fig. $9 \mathrm{BBF}$ at work for solving M1 problem instance, the numbers on the final result shows the placement order of corresponding rectangle.

Figure 9 shows the final result obtained by employing BBF to the M1 problem instance. After BBF starts its execution, $9 \times 1$ is placed as it is into the vertical niche (to the bottom-left-most of the strip) due to the choices of policies in the 7tuple $\left\langle\right.$ Enabled, $\left.{ }^{*}, \mathrm{eVH},{ }^{*},{ }^{*},{ }^{*},{ }^{*}\right\rangle$, and since this rectangle fits exactly into the vertical gap having a width of 9 . Then, both vertical niche and horizontal gap data structures are updated. Since no exact fitting rectangle can be found, a best fitting rectangle is searched in the next step. First, possibility of finding a fitting rectangle for the horizontal gap is explored due to the bHV policy. Moreover, 
based on the policy FP, the tallest rectangle is chosen for placement from the remaining rectangles in the list, that is $8 \times 1$. This piece is placed next to the tallest neighbour, since the placement policy is TN. The sides of the strip are assumed to have an infinite height. Therefore, $8 \times 1$ rectangle is rotated and placed next to the right side of the strip. Again, both vertical niche and horizontal gap data structures are updated. In a similar manner, the first $4 \times 1$ is rotated and placed next to the $9 \times 1$ rectangle. After the data structures are updated, the vertical niche has a width of 5 and a height of 1, whereas the lowest horizontal gap has a width of 1 . An exact fitting rectangle can not be found for the vertical niche, but an exact fitting rectangle can be found for the horizontal niche, that is the rotated version of $4 \times 1$. After this item is placed, BBF continues its execution in the same way for the remaining rectangles. Table 2 shows all the steps that leads to the final result provided in Figure 9. Notice that this result is the optimal result for the given problem instance.

Table 2 Execution order of BBF stages after pre-processing generating the final placement shown in Figure 9 under ordered the policy combination <Enabled, TRE, eVH, FP, WR, bHV, TN>.

\begin{tabular}{|c|c|c|c|c|c|c|c|c|}
\hline \multirow[b]{2}{*}{$\begin{array}{l}\text { execution } \\
\text { order }\end{array}$} & \multirow[b]{2}{*}{$\begin{array}{l}\text { last stage } \\
\text { executed }\end{array}$} & \multirow[b]{2}{*}{$\begin{array}{c}\text { placed } \\
\text { rectangle }\end{array}$} & \multirow[b]{2}{*}{ orientation } & \multicolumn{3}{|c|}{ vertical niche } & \multicolumn{2}{|c|}{$\begin{array}{l}\text { horizontal } \\
\text { lowest gap }\end{array}$} \\
\hline & & & & $\begin{array}{l}(x, y) \\
\text { coord. }\end{array}$ & width & height & $\begin{array}{l}(x, y) \\
\text { coord. }\end{array}$ & width \\
\hline 1 & stage\#1b & $9 \times 1$ & asis & $(1,0)$ & 9 & 3 & $(1,0)$ & 3 \\
\hline 2 & stage\#2a & $8 \times 1$ & Rotated & $(1,0)$ & 9 & 2 & $(1,0)$ & 2 \\
\hline 3 & stage\#2a & $4 \times 1$ & Rotated & $(1,4)$ & 5 & 1 & $(2,0)$ & 1 \\
\hline 4 & stage\#1a & $4 \times 1$ & Rotated & $(1,4)$ & 5 & 2 & $(1,4)$ & 2 \\
\hline 5 & stage\#2a & $4 \times 1$ & Rotated & $(1,8)$ & 1 & 1 & $(2,4)$ & 1 \\
\hline 6 & stage\#1a & $4 \times 1$ & Rotated & - & - & - & - & - \\
\hline
\end{tabular}

\subsection{Running time complexity issues}

All bottom left heuristics run in $\mathrm{O}\left(n^{2}\right)$ time in the worst case for placing $n$ rectangles, assuming an efficient implementation. The worst case running time complexity of the $\mathrm{BF}$ implementation as described in Burke, Kendall and Whitwell (2004) is $\mathrm{O}\left(n \lg n+n^{2}+W n\right)$, where $W$ is the width of the strip. The term $c_{1} n \lg n$ arises due to the sorting of rectangles during the pre-processing phase and tower elimination at the post processing phase. The term $c_{2} n^{2}$ is due to the search for finding the best fitting rectangle to the lowest horizontal gap at each step. Finally, maintaining the skyline and the lowest gap takes $c_{3} W n$ time. The 
representation of the skyline is improved in Burke, Kendall and Whitwell (2006) by a linked list structure, eliminating this term.

A given implementation of an approach can be further improved by using more appropriate data structures. The pseudo-code provided in Figure 6 hides implementation details. BBF is implemented in a similar fashion to the previous implementation of BF for the experiments. In the worst case, the running time of both $\mathrm{BF}$ and $\mathrm{BBF}$ implementations are the same; $\mathrm{O}\left(n^{2}\right)$. The execution time of $\mathrm{BBF}$ for each problem instance used during the experiments is provided in Section 3 to illustrate this phenomenon. Imahori and Yagiura (2009) in their recent study show that the best fit heuristic is a $\Theta(\alpha)$-approximation algorithm, where $\alpha^{\alpha}=n$, $\alpha>0$ and $n$ is the number of rectangles to be placed. The same reasoning provided in their study also applies to the bidirectional best fit heuristic. As the BBF heuristic covers all policies that the best fit heuristic does and even more, it follows that the bidirectional best fit heuristic is also a $\Theta(\alpha)$-approximation algorithm. Moreover, Imahori and Yagiura (2009) describe an implementation in which the running time of the $\mathrm{BF}$ heuristic is improved to $\mathrm{O}(n \log n)$.

\section{Computational results}

\subsection{Benchmark problems and performance evaluation criteria}

BBF is experimented on a set of well known benchmark problems gathered from the previous studies in the literature for comparison. The main properties of these problems are summarized in Table 3. Jakobs (1996) data set has two small problem instances with known optimum heights. Burke, Kendall and Whitwell (2004) data set contains thirteen randomly generated problems. Valenzuela and Wang (2001) data set contains twelve problems, where rectangles have floating point values for each dimension. Half of the problems include rectangles having similar sizes, while the size of the rectangles in the other half differ vastly, labelled as $\mathrm{N}$ (Nice) and $\mathrm{P}$ (Path) problems, respectively. Floating point data is converted into integer values using a scaling factor, determined by some precision. The problems in the Hopper and Turton (2001a) data set are grouped in seven categories. Three problems in each category have the same optimal height and sheet dimensions, while the number of rectangles might differ. Hopper (2000) introduces guillotine and non-guillotine data sets, each containing seven 
categories of five problem instances. During the computational experiments, nonguillotine zero-waste data set having 35 total problem instances is used. Ramesh Babu and Ramesh Babu (1999) present a single problem instance. Pinto and Oliveira (2005) data set consists of large problem instances ranging from 50 to 15,000 rectangular pieces. The BBF algorithm is implemented using Java. The experiments are performed on a Windows computer with an Intel Core 21.86 $\mathrm{GHz}$ CPU and 2GB RAM.

Table 3 The properties of the first class of benchmark problems used during the experiments

\begin{tabular}{|c|c|c|c|c|}
\hline Data source & $\operatorname{Problem}(s)$ & $\begin{array}{l}\text { Number of } \\
\text { rectangles }\end{array}$ & $\begin{array}{c}\text { Optimal } \\
\text { height }\end{array}$ & Sheet width \\
\hline \multirow[t]{7}{*}{ Pinto and Oliveira (2005) } & PO1 & 50 & \multirow{7}{*}{600} & \multirow{7}{*}{400} \\
\hline & $\mathrm{PO} 2$ & 100 & & \\
\hline & PO3 & 500 & & \\
\hline & PO4 & 1,000 & & \\
\hline & PO5 & 5,000 & & \\
\hline & PO6 & 10,000 & & \\
\hline & PO7 & 15,000 & & \\
\hline \multirow{13}{*}{$\begin{array}{l}\text { Burke, Kendall and } \\
\text { Whitwell (2004) }\end{array}$} & N1 & 10 & 40 & 40 \\
\hline & $\mathrm{N} 2$ & 20 & 50 & \multirow{2}{*}{30} \\
\hline & N3 & 30 & 50 & \\
\hline & N4 & 40 & 80 & 80 \\
\hline & N5 & 50 & 100 & 100 \\
\hline & N6 & 60 & 100 & 50 \\
\hline & N7 & 70 & 100 & 80 \\
\hline & N8 & 80 & 80 & 100 \\
\hline & N9 & 100 & 150 & 50 \\
\hline & N10 & 200 & 150 & 70 \\
\hline & N11 & 300 & 150 & \\
\hline & N12 & 500 & 300 & 100 \\
\hline & N13 & 3,152 & 960 & 640 \\
\hline \multirow[t]{6}{*}{ Valenzuela and Wang (2001) } & VN1, VP1 & 25 & \multirow{6}{*}{100} & \multirow{6}{*}{100} \\
\hline & $\mathrm{VN} 2, \mathrm{VP} 2$ & 50 & & \\
\hline & VN3, VP3 & 100 & & \\
\hline & VN4, VP4 & 200 & & \\
\hline & VN5, VP5 & 500 & & \\
\hline & VN6, VP6 & 1,000 & & \\
\hline Hopper and Turton (2001a) & C1P1 & 16 & 20 & 20 \\
\hline
\end{tabular}




\begin{tabular}{|c|c|c|c|c|c|}
\hline & \multirow[t]{2}{*}{$\mathrm{C} 1$} & $\mathrm{C} 1 \mathrm{P} 2$ & 17 & & \\
\hline & & $\mathrm{C} 1 \mathrm{P} 3$ & 16 & & \\
\hline & \multirow[t]{2}{*}{$\mathrm{C} 2$} & $\begin{array}{l}\text { C2-P1, P2, } \\
\text { P3 }\end{array}$ & 25 & 15 & 40 \\
\hline & & C3P1 & 28 & & \\
\hline & \multirow[t]{2}{*}{$\mathrm{C} 3$} & $\mathrm{C} 3 \mathrm{P} 2$ & 29 & 30 & 60 \\
\hline & & $\mathrm{C} 3 \mathrm{P} 3$ & 28 & & \\
\hline & \multirow[t]{2}{*}{$\mathrm{C} 4$} & $\begin{array}{l}\text { C4-P1, P2, } \\
\text { P3 }\end{array}$ & 49 & 60 & 60 \\
\hline & & C5P1 & 72 & & \\
\hline & \multirow[t]{2}{*}{$\mathrm{C} 5$} & $\mathrm{C} 5 \mathrm{P} 2$ & 73 & 90 & 60 \\
\hline & & C5P3 & 72 & & \\
\hline & \multirow[t]{2}{*}{ C6 } & $\begin{array}{l}\text { C6-P1, P2, } \\
\text { P3 }\end{array}$ & 97 & 120 & 80 \\
\hline & & C7P1 & 196 & & \\
\hline & \multirow[t]{2}{*}{$\mathrm{C} 7$} & $\mathrm{C} 7 \mathrm{P} 2$ & 197 & 240 & 160 \\
\hline & & $\mathrm{C} 7 \mathrm{P} 3$ & 196 & & \\
\hline \multirow{7}{*}{\multicolumn{2}{|c|}{$\begin{array}{l}\text { Hopper (2000) } \\
\text { Explanation: There are } 5 \\
\text { problem instances in each } \\
\text { problem category }\end{array}$}} & H1 & 17 & & \\
\hline & & $\mathrm{H} 2$ & 25 & & \\
\hline & & $\mathrm{H} 3$ & 29 & & \\
\hline & & $\mathrm{H} 4$ & 49 & 200 & 200 \\
\hline & & H5 & 73 & & \\
\hline & & H6 & 97 & & \\
\hline & & $\mathrm{H} 7$ & 197 & & \\
\hline \multicolumn{2}{|l|}{$\begin{array}{l}\text { Ramesh Babu and Ramesh } \\
\text { Babu (1999) }\end{array}$} & $\mathrm{RB}$ & 50 & 375 & 1000 \\
\hline \multirow{2}{*}{\multicolumn{2}{|c|}{ Jakobs (1996) }} & $\mathrm{J} 1$ & 25 & \multirow{2}{*}{15} & \multirow{2}{*}{40} \\
\hline & & $\mathrm{J} 2$ & 50 & & \\
\hline
\end{tabular}

All benchmark problem instances, Packer tool provided by Can Başaran to view the placements and the Java code of the heuristic will be available at http://cs.nott.ac.uk/ exo/research/SPP. Two performance evaluation measures will be used for comparing approaches:

- how much improvement an approach provides over another one

- considering the best (or an average) solution, the distance to the optimum (or lower bound).

The percentage improvement (\%-imprv.) that a heuristic\#1 provides over a heuristic\#2 is computed using the following formula:

$\%$-imprv. $=100 \times\left(\right.$ resultant_height $t_{2}-$ resultant_height 1$) /$ resultant_height $_{2}$ 
where resultant_height $t_{j}$ is the best height obtained using the heuristic\#j. A value of 0 indicates that both heuristics generate the same result. A negative value is not reported as it means that heuristic\#1 does not improve the solution generated by heuristic\#2. The optimal solution or a lower bound for each problem instance is known. Hence, \%-gap is used to measure how much a solution obtained by using a heuristic deviates from the optimal solution (or a lower bound) denoted as opt_height as defined in Equation (3). A lower \%-gap indicates a better performance.

$\%-g a p=100 \times($ resultant_height - opt_height $) /$ opt_height

\subsection{Policy tests}

If one of the policies as described in Section 2.2 and summarized in Table 1 is left out, then the result obtained for at least one of the problem instances worsens as illustrated in Table 4. Each policy used within BBF is essential. Yet, it is observed that the same solution might be obtained with different policy combinations. As an example, two optimal placements obtained from data C1P1 using BFF are shown in Figure 10. Note that, Burke, Kendall and Whitwell (2004) already showed that different policies for placement into the horizontal gap yield different results.

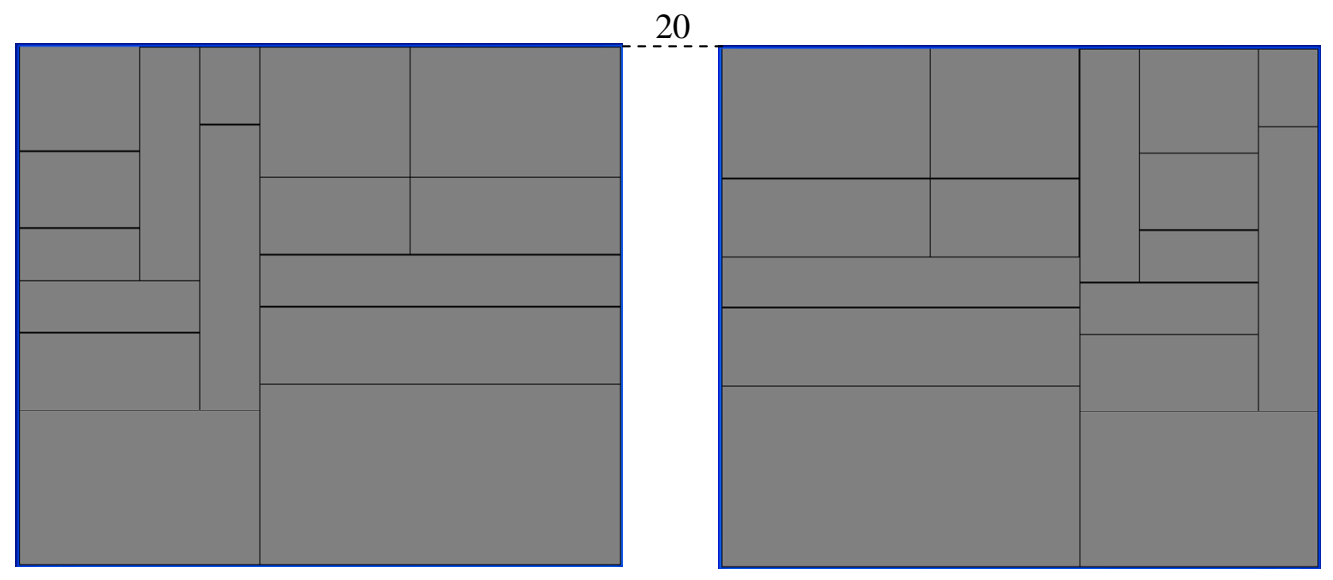

Fig. 10 Two different optimal solutions generated by BFF from data C1P1 using different policy combinations.

Table 4 Height 2 is the result obtained for a given RF-SPP instance after applying the BBF algorithm by excluding the corresponding policy assignment. Height 1 is the result obtained whenever all policies are used 


\begin{tabular}{cllrrr}
\hline Policy id. & Assignment & Problem & Optimal & Height1 & Height2 \\
\hline \multirow{4}{*}{1} & Enabled & N7 & 100 & 106 & 109 \\
& Disabled & N2 & 50 & 52 & 53 \\
& TRE & C4P2 & 60 & 62 & 63 \\
2 & NRE & N10 & 150 & 151 & 152 \\
& eHV & C7P3 & 240 & 244 & 245 \\
3 & eVH & N9 & 150 & 152 & 160 \\
& BP & VN2 & 1000 & 1080 & 1085 \\
4 & FP & N12 & 300 & 303 & 305 \\
& FH & N11 & 150 & 151 & 152 \\
5 & WR & N4 & 80 & 82 & 83 \\
& noVB & C6P2 & 120 & 121 & 123 \\
& bHV & N1 & 40 & 40 & 43 \\
6 & bVH & N8 & 80 & 82 & 83 \\
\hline
\end{tabular}

\subsection{Comparison of BBF to some previous heuristics}

Table 5(a) presents the experimental results from using Zhang, Kang and Deng (2006) heuristic, denoted as $H R$, bottom-left, bottom-left-fill, their variants and our new approach over Hopper and Turton (2001a) data set. The proposed heuristic either improves the solutions or generates a tie for all problem instances obtained from the previous heuristics. BBF finds the optimal result for data C1P1 and C2P3. It turns out to be the best approach with an average \%-gap of $\% 2.95$ over the problem instances. BBF performs still better as compared to the bottomleft heuristic as the problem size increases (Table 5(b)) over the Pinto and Oliveira (2005) data. Student's paired t-test over \%-gaps indicates that the performance variation between $\mathrm{BFF}$ and a blind heuristic is statistically significant within a confidence interval of $99.99 \%$ in both cases. The computational experiments over the Hopper data as presented in Table 5(c) shows that the average performances of $\mathrm{BBF}$ and $\mathrm{BLD}^{*}$ are comparable based on the best results, moreover, BBF outperforms BLD* heuristic (Lesh et al., 2005) in 4 data sets out of 7. Jakobs (1996) reports that applying BL heuristic to J1 and J2 after sorting the rectangles in decreasing widths yields a gap of $\% 40$ for each, while BBF generates a gap of \%6.7 for each one of these small problems (Table 5(d)).

Table 5 Comparison of the bidirectional best fit heuristic to (a) $H R$ heuristic of Zhang, Kang and Deng (2006) and a variety of bottom-left heuristics and the best fit heuristic over Hopper and Turton data set based on \%-gap. The bold entries mark the best performing approach(es). $H$ and $W$ indicate that the list of rectangles used as input is sorted with respect to their widths and heights, respectively in decreasing order $(D)$, (b) the bottom left heuristic as reported in Alvarez-Valdes et al. (2008) over Pinto and Oliveira data set, (c) BLD* of Lesh et al. (2005) over Hopper data set run 
in 60 seconds and 3600 seconds. The bold entries mark the best performing heuristic(s) (d) BLDW as reported in Jakobs (1996). The execution time of BBF for each problem is provided in (b), (c) and (d).

(a)

\begin{tabular}{|c|c|c|c|c|c|c|c|c|c|}
\hline label & $\begin{array}{c}\begin{array}{c}\text { no. of } \\
\text { shapes }\end{array} \\
\end{array}$ & $\mathrm{BL}$ & BL-DW & BL-DH & BLF & BLF-DW & BLF-DH & $\mathrm{BBF}$ & HR \\
\hline C1P1 & 16 & 45 & 30 & 15 & 30 & 10 & 10 & 0 & \\
\hline $\mathrm{C} 1 \mathrm{P} 2$ & 17 & 40 & 20 & 10 & 35 & 15 & 10 & 5 & \\
\hline C1P3 & 16 & 35 & 20 & 5 & 25 & 15 & 5 & 5 & \\
\hline $\mathrm{C} 1$ & avr & 40.0 & 23.3 & 10.0 & 30.0 & 13.3 & 8.3 & 3.3 & 8.3 \\
\hline $\mathrm{C} 2 \mathrm{P} 1$ & 25 & 53 & 13 & 13 & 47 & 13 & 13 & 6.7 & \\
\hline $\mathrm{C} 2 \mathrm{P} 2$ & 25 & 80 & 27 & 73 & 73 & 20 & 73 & 6.7 & \\
\hline $\mathrm{C} 2 \mathrm{P} 3$ & 25 & 67 & 27 & 13 & 47 & 20 & 13 & 0 & \\
\hline $\mathrm{C} 2$ & avr & 66.7 & 22.3 & 33.0 & 55.7 & 17.7 & 33.0 & 4.4 & 4.5 \\
\hline C3P1 & 28 & 40 & 10 & 10 & 37 & 10 & 10 & 0 & \\
\hline C3P2 & 29 & 43 & 20 & 10 & 50 & 13 & 6.7 & 10 & \\
\hline C3P3 & 28 & 40 & 17 & 13 & 33 & 13 & 13 & 3.3 & \\
\hline $\mathrm{C} 3$ & avr & 41.0 & 15.7 & 11.0 & 40.0 & 12.0 & 9.9 & 4.4 & 6.7 \\
\hline C4P1 & 49 & 32 & 17 & 12 & 25 & 10 & 10 & 3.3 & \\
\hline $\mathrm{C} 4 \mathrm{P} 2$ & 49 & 37 & 22 & 13 & 25 & 5 & 5 & 3.3 & \\
\hline C4P3 & 49 & 30 & 22 & 6.7 & 27 & 10 & 5 & 1.7 & \\
\hline $\mathrm{C} 4$ & avr & 33.0 & 20.33 & 10.57 & 25.67 & 8.33 & 6.7 & 2.8 & 2.2 \\
\hline C5P1 & 72 & 27 & 16 & 4.4 & 20 & 5.6 & 4.4 & 1.1 & \\
\hline C5P2 & 73 & 32 & 18 & 10 & 23 & 6.7 & 5.6 & 2.2 & \\
\hline C5P3 & 72 & 30 & 13 & 7.8 & 21 & 5.6 & 4.4 & 1.1 & \\
\hline C5 & avr & 29.7 & 15.7 & 7.4 & 21.3 & 6.0 & 4.8 & 1.5 & 1.9 \\
\hline C6P1 & 97 & 33 & 22 & 8.3 & 20 & 5 & 5 & 1.7 & \\
\hline C6Р2 & 97 & 39 & 25 & 8.3 & 18 & 4.2 & 2.5 & 0.8 & \\
\hline C6P3 & 97 & 34 & 18 & 9.2 & 21 & 4.2 & 6.7 & 1.7 & \\
\hline C6 & avr & 35.3 & 21.7 & 8.6 & 19.7 & 4.5 & 4.7 & 1.4 & 2.5 \\
\hline C7P1 & 196 & 22 & 16 & 5 & 15 & 4.6 & 3.8 & 1.2 & \\
\hline C7P2 & 197 & 41 & 19 & 10 & 20 & 3.3 & 2.9 & 1.7 & \\
\hline C7P3 & 196 & 31 & 17 & 7.1 & 17 & 2.9 & 3.8 & 1.7 & \\
\hline $\mathrm{C} 7$ & avr & 31.3 & 17.3 & 7.4 & 17.3 & 3.6 & 3.5 & 1.5 & 1.8 \\
\hline
\end{tabular}

(b)

\begin{tabular}{rrrrrr}
\hline label & $\begin{array}{r}\text { no. of } \\
\text { shapes }\end{array}$ & Optimum & $\begin{array}{r}\text { Time } \\
(\mathrm{sec})\end{array}$ & BBF & BL \\
\hline PO1 & 50 & & 0.25 & $\mathbf{7 . 2}$ & 12.3 \\
PO2 & 100 & & 0.61 & $\mathbf{7 . 5}$ & 13.2 \\
PO3 & 500 & & 6.23 & $\mathbf{3 . 0}$ & 15.3 \\
PO4 & 1,000 & 600 & 17.87 & $\mathbf{0 . 0}$ & 15.0 \\
PO5 & 5,000 & & 413.94 & $\mathbf{0 . 0}$ & 14.5 \\
PO6 & 10,000 & & 1780.91 & $\mathbf{0 . 0}$ & 13.5 \\
PO7 & 15,000 & & 4237.50 & $\mathbf{0 . 0}$ & 10.0 \\
\hline & & & avr & 2.5 & 13.4 \\
\cline { 3 - 5 } & & &
\end{tabular}

(c)

\begin{tabular}{ccclrlrrrr}
\hline & no. of & & \multicolumn{2}{l}{ BBF } & & \multicolumn{3}{l}{ BLD* } \\
label & shapes & optimum & Mean time (sec) & Mean & Best & & $60 \mathrm{~s}$ & $3600 \mathrm{~s}$ \\
\hline H1 & 17 & & 216.6 & 8.3 & 6.0 & & 6.0 & $\mathbf{4 . 5}$
\end{tabular}




\begin{tabular}{|c|c|c|c|c|c|c|c|}
\hline $\mathrm{H} 2$ & 25 & & 216 & 8.0 & 6.5 & 6.4 & 4.7 \\
\hline $\mathrm{H} 3$ & 29 & \multirow{5}{*}{200} & 214.6 & 7.3 & 5.0 & 6.0 & 4.6 \\
\hline $\mathrm{H} 4$ & 49 & & 209 & 4.5 & 2.5 & 5.1 & 3.9 \\
\hline H5 & 73 & & 209.4 & 4.7 & 3.5 & 4.6 & 4.0 \\
\hline H6 & 97 & & 205.8 & 2.9 & 2.5 & 4.0 & 3.0 \\
\hline H7 & 197 & & 203.2 & 1.6 & 1.5 & 2.3 & 1.8 \\
\hline & & & & avr & 3.9 & 4.9 & 3.8 \\
\hline
\end{tabular}

(d)

\begin{tabular}{rcccrc}
\hline label & no. of shapes & optimum & Time (sec) & BBF & BL-DW \\
\hline J1 & 25 & 15 & 0.06 & $\mathbf{6 . 7}$ & 40 \\
J2 & 50 & & 0.11 & $\mathbf{6 . 7}$ & 40 \\
\hline
\end{tabular}

\subsection{Comparison of BBF to BF}

Table 6 presents a performance comparison of BBF to BF. In 32 problem instances, the improved heuristic outperforms the best fit heuristic. In only 3 problem instances $\{\mathrm{VN} 4, \mathrm{VP} 3, \mathrm{VP} 4\}$, the best fit heuristic has a better performance, while in the rest of the problems, there is a tie. There are inconsistencies between the best fit heuristic results in Burke et al. (2004) and Burke et al. (2006) for the Valenzuela and Wang (2001) problem instances. The reason for this phenomenon and getting better results from BF for $\{\mathrm{VN} 4, \mathrm{VP}\}$, VP4 $\}$ as compared to BBF appears to be due to the conversion from floating point to integer data. For future comparisons, the exact problem instances used during the experiments are publicly available (see Section 3.1). Although the solution qualities generated by $\mathrm{BFF}$ and $\mathrm{BF}$ are the same for data $\mathrm{C} 7 \mathrm{P} 2$ and $\mathrm{RB}$, the resultant placements demonstrated in Figure 11 are different than the ones as presented in Burke Kendall, and Whitwell (2004). BBF obtains optimal solutions for four problem instances $\{\mathrm{N} 1, \mathrm{C} 1 \mathrm{P} 1, \mathrm{C} 2 \mathrm{P} 3, \mathrm{C} 3 \mathrm{P} 1\}$. The average $\%$-gap over all the problem instances excluding M1 for BBF is 3.49, while it is 5.45 for BF. Student's paired t-test based on \%-gaps shows that this performance variation is also statistically significant within a confidence interval of $99.99 \%$.

Table 6 Comparison of the bidirectional best fit heuristic (BBF) to the best fit heuristic (BF) results provided in Burke et al. (2004) based on \%-gap and \%-impr. The execution time of BBF for each problem is also provided. The bold entries mark the best performing heuristic(s).

\begin{tabular}{crrrrrrrr}
\hline \multirow{2}{*}{ label } & $\begin{array}{r}\text { number } \\
\text { of shapes }\end{array}$ & $\begin{array}{r}\text { optimal } \\
\text { height }\end{array}$ & $\begin{array}{r}\text { BBF } \\
\text { result }\end{array}$ & $\begin{array}{r}\text { BF } \\
\text { result }\end{array}$ & $\begin{array}{r}\text { Time } \\
(\mathrm{sec})\end{array}$ & $\begin{array}{r}\text { BBF } \\
\% \text {-gap }\end{array}$ & $\begin{array}{r}\text { \%F-gap } \\
\text { \% }\end{array}$ & $\begin{array}{c}\text { impr. } \\
\text { M1 }\end{array}$ \\
\hline
\end{tabular}




\begin{tabular}{|c|c|c|c|c|c|c|c|c|}
\hline $\mathrm{N} 1$ & 10 & 40 & 40 & 45 & 0.03 & 0.00 & 12.50 & 11.11 \\
\hline $\mathrm{N} 2$ & 20 & 50 & 52 & 53 & 0.02 & 4.00 & 6.00 & 1.89 \\
\hline N3 & 30 & 50 & 52 & 52 & 0.05 & 4.00 & 4.00 & 0.00 \\
\hline $\mathrm{N} 4$ & 40 & 80 & 82 & 83 & 0.09 & 2.50 & 3.75 & 1.20 \\
\hline N5 & 50 & 100 & 104 & 105 & 0.14 & 4.00 & 5.00 & 0.95 \\
\hline N6 & 60 & 100 & 102 & 103 & 0.16 & 2.00 & 3.00 & 0.97 \\
\hline N7 & 70 & 100 & 106 & 107 & 0.22 & 6.00 & 7.00 & 0.93 \\
\hline N8 & 80 & 80 & 82 & 84 & 0.28 & 2.50 & 5.00 & 2.38 \\
\hline N9 & 100 & 150 & 152 & 152 & 0.33 & 1.33 & 1.33 & 0.00 \\
\hline N10 & 200 & 150 & 151 & 152 & 1.13 & 0.67 & 1.33 & 0.66 \\
\hline N11 & 300 & 150 & 151 & 152 & 2.14 & 0.67 & 1.33 & 0.66 \\
\hline N12 & 500 & 300 & 303 & 306 & 5.95 & 1.00 & 2.00 & 0.98 \\
\hline N13 & 3,152 & 960 & 964 & 964 & 163.42 & 0.42 & 0.42 & 0.00 \\
\hline C1P1 & 16 & 20 & 20 & 21 & 0.02 & 0.00 & 5.00 & 4.76 \\
\hline $\mathrm{C} 1 \mathrm{P} 2$ & 17 & 20 & 21 & 22 & 0.02 & 5.00 & 10.00 & 4.55 \\
\hline $\mathrm{C} 1 \mathrm{P} 3$ & 16 & 20 & 21 & 24 & 0.02 & 5.00 & 20.00 & 12.50 \\
\hline $\mathrm{C} 2 \mathrm{P} 1$ & 25 & 15 & 16 & 16 & 0.05 & 6.67 & 6.67 & 0.00 \\
\hline $\mathrm{C} 2 \mathrm{P} 2$ & 25 & 15 & 16 & 16 & 0.03 & 6.67 & 6.67 & 0.00 \\
\hline $\mathrm{C} 2 \mathrm{P} 3$ & 25 & 15 & 15 & 16 & 0.03 & 0.00 & 6.67 & 6.25 \\
\hline C3P1 & 28 & 30 & 30 & 32 & 0.05 & 0.00 & 6.67 & 6.25 \\
\hline $\mathrm{C} 3 \mathrm{P} 2$ & 29 & 30 & 33 & 34 & 0.05 & 10.00 & 13.33 & 2.94 \\
\hline C3P3 & 28 & 30 & 31 & 33 & 0.05 & 3.33 & 10.00 & 6.06 \\
\hline C4P1 & 49 & 60 & 62 & 63 & 0.11 & 3.33 & 5.00 & 1.59 \\
\hline $\mathrm{C} 4 \mathrm{P} 2$ & 49 & 60 & 62 & 62 & 0.11 & 3.33 & 3.33 & 0.00 \\
\hline C4P3 & 49 & 60 & 61 & 62 & 0.11 & 1.67 & 3.33 & 1.61 \\
\hline C5P1 & 72 & 90 & 91 & 93 & 0.19 & 1.11 & 3.33 & 2.15 \\
\hline $\mathrm{C} 5 \mathrm{P} 2$ & 73 & 90 & 92 & 92 & 0.19 & 2.22 & 2.22 & 0.00 \\
\hline $\mathrm{C} 5 \mathrm{P} 3$ & 72 & 90 & 91 & 93 & 0.19 & 1.11 & 3.33 & 2.15 \\
\hline C6P1 & 97 & 120 & 122 & 123 & 0.33 & 1.67 & 2.50 & 0.81 \\
\hline C6P2 & 97 & 120 & 121 & 122 & 0.31 & 0.83 & 1.67 & 0.82 \\
\hline C6P3 & 97 & 120 & 122 & 124 & 0.38 & 1.67 & 3.33 & 1.61 \\
\hline C7P1 & 196 & 240 & 243 & 247 & 1.20 & 1.25 & 2.92 & 1.62 \\
\hline C7P2 & 197 & 240 & 244 & 244 & 1.05 & 1.67 & 1.67 & 0.00 \\
\hline C7P3 & 196 & 240 & 244 & 245 & 1.14 & 1.67 & 2.08 & 0.41 \\
\hline VN1 & 25 & 1000 & 1073 & 1074 & 0.22 & 7.30 & 7.40 & 0.09 \\
\hline VN2 & 50 & 1000 & 1080 & 1085 & 0.48 & 8.00 & 8.50 & 0.46 \\
\hline VN3 & 100 & 1000 & 1069 & 1070 & 1.14 & 6.90 & 7.00 & 0.09 \\
\hline VN4 & 200 & 1000 & 1056 & 1053 & 2.97 & 5.60 & 5.30 & - \\
\hline VN5 & 500 & 1000 & 1031 & 1035 & 12.02 & 3.10 & 3.50 & 0.39 \\
\hline VN6 & 1000 & 1000 & 1036 & 1037 & 42.20 & 3.60 & 3.70 & 0.10 \\
\hline VP1 & 25 & 1000 & 1093 & 1101 & 0.22 & 9.30 & 10.10 & 0.73 \\
\hline
\end{tabular}




\begin{tabular}{rrrrrrrrr} 
VP2 & 50 & 1000 & $\mathbf{1 0 7 4}$ & 1138 & 0.44 & 7.40 & 13.80 & 5.62 \\
VP3 & 100 & 1000 & 1080 & $\mathbf{1 0 7 3}$ & 1.05 & 8.00 & 7.30 & - \\
VP4 & 200 & 1000 & 1053 & $\mathbf{1 0 4 1}$ & 2.61 & 5.30 & 4.10 & - \\
VP5 & 500 & 1000 & $\mathbf{1 0 3 1}$ & 1037 & 10.06 & 3.10 & 3.70 & 0.58 \\
VP6 & 1000 & 1000 & $\mathbf{1 0 2 6}$ & 1028 & 31.56 & 2.60 & 2.80 & 0.19 \\
\hline RB & 50 & 375 & $\mathbf{4 0 0}$ & $\mathbf{4 0 0}$ & 0.36 & 6.67 & 6.67 & 0.00 \\
\hline
\end{tabular}

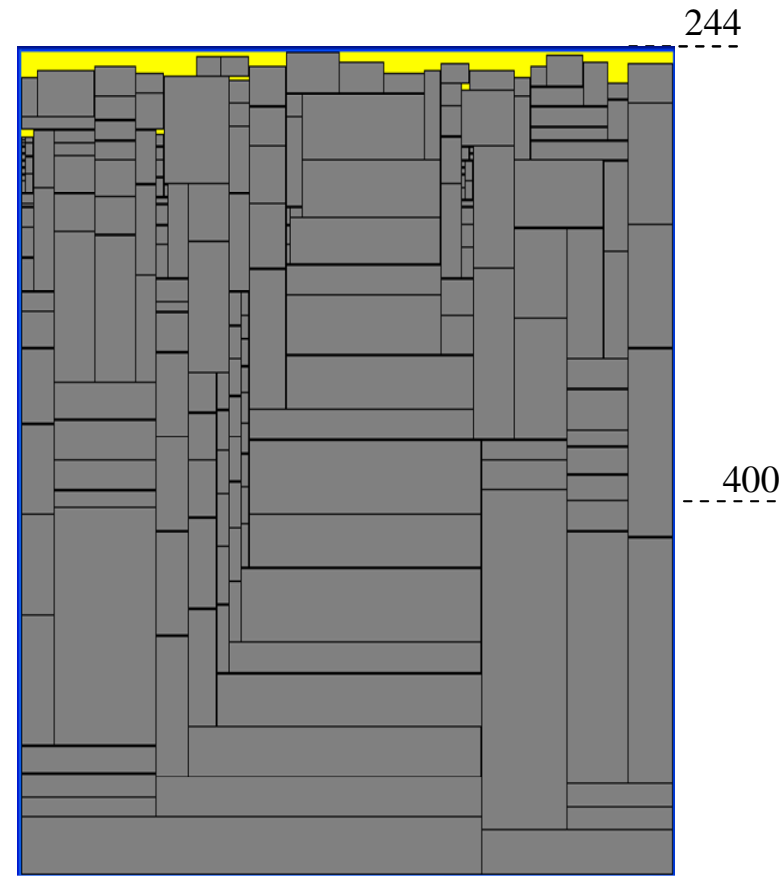

(a)

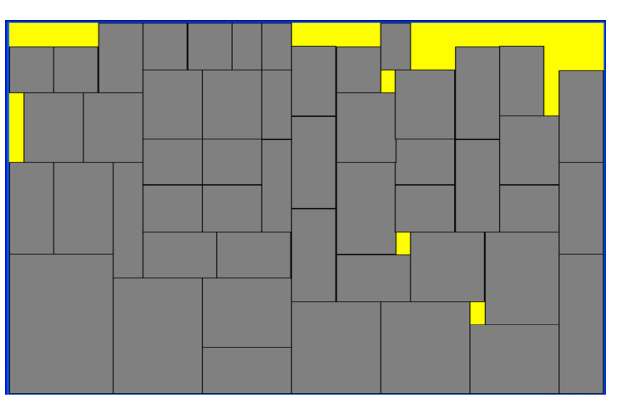

(b)

Fig. 11 The solutions generated by BBF from data (a) C7P2 and (b) RB

\subsection{Comparison of BBF to the meta-heuristics}

Table 7 presents performance comparison of BBF to the genetic algorithm (GA), simulated annealing (SA) and GRASP meta-heuristics. Jakobs (1996) searched for an optimal ordering of rectangles using a GA. A candidate solution requires a decoder that will place all rectangles in the given order onto the strip and the author used BL (GA+BL). Liu and Teng (1999) proposed improved BL heuristic as a decoder and utilised in their GA $(\mathrm{GA}+\mathrm{iBL})$. The results show that BBF performs better than $\mathrm{GA}+\mathrm{BL}$ and delivers a comparable performance to $\mathrm{GA}+\mathrm{iBL}$ for J1 and J2 (Table 7(a)). Hopper and Turton (2001a) and Burke et al. (2004) provided a GA and SA that utilise BLF as a decoder (op+BLF). Beltrán et al. (2004) first applied a GRASP for obtaining an optimal ordering of rectangles using $\mathrm{BL}$ as a decoder and then improved the solution using a variable 
neighbourhood search (vGRASP). Bortfeldt (2006) presented a genetic algorithm based on a layered approach (SPGAL). Alvarez-Valdes et al. (2008) obtained one of the best results in literature by iteratively progressing towards a better solution through constructive and improvement phases using a GRASP approach (rGRASP) in a multi-start setting. Imahori et al. (2005) employed iterated local search for solving RF-SPPs. Their approach perturbs a candidate solution of sequence of rectangles and attempts to improve it at each step. The authors used a different performance evaluation scheme in their study, for this reason an indirect and relative comparison is provided as follows. According to their results, iterated local search improves SA+BLF by $\% 0.5$ based on their evaluation scheme, whereas BBF performs slightly better with a \%0.8 improvement over SA+BLF based on average \%-gap considering Hopper and Turton problem instances. The results also show that BBF performs better than six older meta-heuristics. On the other hand, two recent meta-heuristic approaches seem to yield better performances, namely; SPGAL and rGRASP as summarized in Table 7(b).

Table 7 Performance comparison of the bidirectional best fit heuristic (BBF) to the metaheuristics based on \%-gap. BL-DW denotes the bottom left heuristic for which the rectangles are sorted in decreasing width.

(a)

\begin{tabular}{rcrrr}
\hline label & BL-DW & BBF & GA+BL & GA+iBL \\
\hline J1 & 40 & $\mathbf{6 . 7}$ & 13.3 & $\mathbf{6 . 7}$ \\
J2 & 40 & $\mathbf{6 . 7}$ & 13.3 & $\mathbf{6 . 7}$ \\
\hline
\end{tabular}

(b)

\begin{tabular}{crrrrrrrrr}
\hline & & \multicolumn{2}{c}{ Hopper } & & \multicolumn{2}{c}{ Burke } & & \\
label & BBF & GA+BLF & SA+BLF & vGRASP & GA+BLF & SA+BLF & SPGAL & rGRASP \\
\hline C1 & 3.33 & 4 & 4 & 14.4 & 1.7 & 1.7 & 1.7 & $\mathbf{0 . 0}$ \\
C2 & 4.44 & 7 & 6 & 17.3 & 6.7 & 6.7 & $\mathbf{0 . 0}$ & $\mathbf{0 . 0}$ \\
C3 & 4.44 & 5 & 5 & 12.9 & 6.7 & 6.7 & 2.2 & $\mathbf{1 . 1}$ \\
C4 & 2.78 & 3 & 3 & 6.8 & 5.0 & 6.1 & $\mathbf{0 . 0}$ & 1.6 \\
C5 & 1.48 & 4 & 3 & 4.5 & 5.6 & 5.2 & $\mathbf{0 . 0}$ & 1.1 \\
C6 & 1.39 & 4 & 3 & 3.6 & 5.3 & 5.3 & 0.3 & $\mathbf{0 . 8}$ \\
C7 & 1.53 & 5 & 4 & 2.9 & 5.6 & 6.0 & 0.3 & $\mathbf{1 . 2}$ \\
\hline avr & 2.77 & 4.57 & 4.00 & 8.92 & 5.20 & 5.36 & $\mathbf{0 . 6 4}$ & 0.84 \\
\hline
\end{tabular}

(c)

\begin{tabular}{rrrrr}
\hline label & no. of shapes & optimum & rGRASP & BBF \\
\hline PO1 & 50 & & $\mathbf{2 . 8 3}$ & 7.17 \\
PO2 & 100 & & $\mathbf{2 . 8 3}$ & 7.50 \\
PO3 & 500 & & $\mathbf{0 . 8 3}$ & 3.00 \\
PO4 & 1,000 & 600 & 0.33 & $\mathbf{0 . 0 0}$ \\
PO5 & 5,000 & & $\mathbf{0 . 0 0}$ & $\mathbf{0 . 0 0}$
\end{tabular}




\begin{tabular}{|c|c|c|c|c|}
\hline PO6 & 10,000 & & 0.00 & 0.00 \\
\hline PO7 & 15,000 & & 0.00 & 0.00 \\
\hline & & avr & 0.98 & 2.52 \\
\hline
\end{tabular}

Burke et al. (2006) experimented with a multistage scheme. BF is executed for a predetermined number of rectangles, and then a meta-heuristic with BLF decoder is invoked. Different meta-heuristics are tested, namely; GA, SA and tabu search (TS). This scheme yielded a better performance as compared to BF. For all data sets in Table 5, excluding M1, BF-GA+BLF, BF-TS+BLF and BF-SA+BLF returns an average gap of $3.61 \%, 3.29 \%$ and $2.86 \%$, respectively. BBF with an average gap of $3.49 \%$ performs slightly better than BF-GA+BLF and slightly worse than the others, yet we can say that it has a comparable performance to these approaches. On the other hand, rGRASP still delivers a superior performance over these benchmarks with an average gap of $1.86 \%$. rGRASP $(0.98 \%)$ has a better average performance as compared to BBF $(2.52 \%)$ for the Pinto-Oliveira data, although BBF obtains the optimal solution for 4 problem instances, while GRASP return an optimal solution for 3 problem instances as shown in Table 7(c). Both approaches seem to deliver a better performance as the size of the problem increases.

BBF can not be compared directly to the hyper-heuristics in Terashima-Marin, Moran-Saavedra, Ross (2005) and Terashima-Marin, Flores-Alvarez, Ross (2005), since the authors preferred a rather rough performance measure which is the minimum number of objects needed to satisfy the packing (cutting) of the demand of pieces to compare their approaches to the others. Both of the approaches generate a similar performance to the Hopper and Turton's best metaheuristic. It is observed from their results that only for a couple of problem instances they can improve over Hopper and Turton's results. On average, the improvements become insignificant. On the other hand, BBF outperforms Hopper and Turton's best meta-heuristics in 6 out of 7 data sets delivering a better average performance (see Table 7(b)). Consequently, it is likely that BBF might outperform these hyper-heuristics, or at least deliver a comparable performance.

\section{Concluding Remarks}

Bidirectional best fit heuristic (BBF) for solving orthogonal rectangular strip packing problems of RF subtype (RF-SPP) is introduced in this paper. The main 
difference of the proposed heuristic from the previous ones is that the niches are maintained and considered for placement in both horizontal and vertical directions. Combining this strategy with a set of useful extended policies, the bidirectional best fit heuristic yields promising results. The performance of BBF is compared to some previous approaches over a set of well known benchmark problem instances with different characteristics, such as the problem size, the size variance of the rectangles, etc. The best fit approach of Burke, Kendall and Whitwell (2004) is reported as one of the best heuristics for solving RF-SPPs. The experimental results indicate that the bidirectional best fit heuristic even improves on the results obtained by them for most of the problem instances.

Although they are more general approaches, SPGAL (Bortfeldt 2006) and GRASPr (Alvarez-Valdes et al. 2008) seem to be the best meta-heuristics for solving SPP variants. The results show that they might perform better than BBF for solving RF-SPPs. With these few exceptions, BBF still performs better than most of the previously proposed meta-heuristics reported in literature. The handicap of meta-heuristics is that a single run might not be sufficient to obtain a good solution due to their stochastic nature. Hence, more execution time might be needed. On the other hand, BBF is a reasonably fast heuristic with a polynomial running time complexity. Its performance can be improved even further using the data structures suggested by Imahori and Yagiura (2009). This is left as a future study.

\section{References}

Alvarez-Valdes, R., Parreno, F. \& Tamarit, J.M. (2008). Reactive GRASP for the strip-packing problem. Computers and Operations Research, 35, 1065-1083.

Baker, B. S., Coffman, E. G., \& Jr Rivest, R. L. (1980). Orthogonal packings in two dimensions. SIAM J on Comput, 9(4), 808-826.

Beasley, J. E. (1985a). An exact two-dimensional non-guillotine cutting tree search procedure. Operations Research, 33(1), 49-64.

Beasley, J. E. (1985b). Algorithms for unconstrained two-dimensional guillotine cutting. $J$ Operations Research Society, 36(4), 297-306.

Beltrán, J. D., Calderón, J. E. , Cabrera, R. J. , Moreno-Pérez, J. A., \& Moreno-Vega, J. M. (2004). GRASP-VNS hybrid for the Strip Packing Problem. Proc. of the workshop on Hybrid Metaheuristics (pp. 79-90).

Bortfeld, A. (2006). A genetic algorithm fot the two-dimensional strip packing problem with rectangular pieces. Eur J Oper Res, 172, 814-837. 
Burke, E., Hart, E., Kendall, G., Newall, J., Ross, P., \& Schulenburg, S. (2003). Hyper-heuristics: An emerging direction in modem search technology. Handbook of Metaheuristics, Kluwer Academic Publishers, 457-474.

Burke, E. K., Kendall, G., \& Whitwell, G. (2004). A new placement heuristic for the orthogonal stock-cutting problem. Operations Research, 52(4), 655-671.

Burke, E. K., Kendall, G., \& Whitwell, G. (2006). Metaheuristic enhancements of the best-fit heuristic for the orthogonal stock cutting problem computer science. Technical Report No NOTTCS-TR-2006-3.

Chazelle, B. (1983). The bottom-left bin-packing heuristic: An efficient implementation. IEEE Transactions on Computers, 32/8, 697-707.

Dagli, C. H., \& Poshyanonda, P. (1997). New approaches to nesting rectangular patterns. Journal of Intelligent Manufacturing, 8, 177-90.

Dowsland, K. A., \& Dowsland, W. B. (1992). Packing Problems. Eur J Oper Res, 56, 2-14.

Garey, M. R., \& Johnson, D. S. (1979). Computers and Intractability. WH Freeman and Company: NY.

Jakobs, S. (1996). On genetic algorithms for the packing of polygons. Eur J Oper Res, 88, 165181.

Hässler, R. W., \& Sweeney, P. E. (1991). Cutting stock problems and solution procedures. Eur J Oper Res, 54, 141-150.

Hinxman, A. I. (1980). The trim loss and assortment problems: A survey. Eur J Oper Res 88, 1, 818.

Hopper, E. (2000). Two-dimensional packing utilising evolutionary algorithms and other metaheuristic methods, PhD thesis, Cardiff University, School of Engineering.

Hopper, E., \& Turton, B. C. H. (2001a). An empirical investigation of metaheuristic and heuristic algorithms for a 2D packing problem. Eur J Oper Res, 128, 34-57.

Hopper, E., \& Turton, B. C. H. (2001b). A review of the application of meta-heuristic algorithms to 2D strip packing problems. Artificial Intelligence Review 257-300.

Kenmochi, M., Imamichi, T., Nonobe, K., Yagiura, M., \& Nagamochi, H. (2007). Exact algorithms for the 2-dimensional strip packing problem with and without rotations. Technical report 2007-005, Dept of Applied Mathematics and Physics, Graduate School of Informatics, Kyoto University, January.

Imahori, S., Yagiura, M., \& Ibaraki, T. (2005). Improved local search algorithms for the rectangle packing problem with general spatial costs. Eur J Oper Res, 167, 48-67.

Imahori, S., Yagiura, M., (2009). The best-fit heuristic for the rectangular strip packing problem: An efficient implementation and the worst-case approximation ratio. Computers \& Operations Research. DOI:10.1016/j.cor.2009.05.008

Lesh, N., Marks, J., McMahon, A., \& Mitzenmacher, M. (2004). Exhaustive approaches to 2D rectangular perfect packings. Information Processing Letters, 90, 7-14.

Lesh, N., Marks, J., McMahon, A., \& Mitzenmacher, M. (2005). New heuristic and interactive approaches to 2D rectangular strip packing. ACM Journal of Experimental Algorithmics, 10, 1-18. 
Liu, D., \& Teng, H. (1999). An improved bottom left algorithm for genetic algorithm of the orthogonal packing of rectangle. Eur J Oper Res, 112, 413-419.

Lodi, A., Martello, S., \& Monaci, M. (2002). Two-dimensional packing problems: a survey. Eur J Oper Res, 141(2), 241-52.

Lodi, A., Martello, S., \& Vigo, D. (1999). Heuristic and metaheuristic approaches for a class of two-dimensional bin packing problems. INFORMS J on Computing;11:345-57.

Martello, S., Monaci, M., \& Vigo, D. (2003). An exact approach to the strip-packing problem. INFORMS J on Computing, 15(3), 310-319.

Martello, S, \& D Vigo (1998). Exact solution of the two-dimensional finite bin packing problem. Management Science, 44, 388-399.

Ozcan, E., Bilgin, B., \& Korkmaz, E. E. (2008). A comprehensive analysis of hyper-heuristics. Intelligent Data Analysis, 12(1), 3-23.

Pinto, E., \& Oliveira, J. F. (2005). Algorithm based on graphs for the non-guillotinable twodimensional packing problem, 2nd ESICUP Meeting, Southampton.

Ramesh Babu, A., \& Ramesh Babu, N. (1999). Effective nesting of rectangular parts in multiple rectangular sheets using genetic and heuristic algorithms. Int J Production Res, 37(7), 16251643.

Sarin, S. C. (1983). Two-dimensional stock cutting problems and solution methodologies ASME Transactions, Journal of Engineering for Industry, 104, 155-160.

Terashima-Marin, H., Moran-Saavedra, A., \& Ross, P. (2005). Forming hyper-heuristics with GAs when solving 2D-regular cutting stock problems. Proceedings of the IEEE Congress on Evolutionary Computation, vol 2 (pp. 1104-1110).

Terashima-Marin, H., Flores-Alvarez, E. J., \& Ross, P. (2005). Hyper-heuristics and Classifier Systems for solving 2D-regular cutting stock problems. Proceedings of the GECCO, vol 2 (pp. 637-643).

Valenzuela, C. L., \& Wang, P. Y. (2001). Heuristics for large strip packing problems with guillotine patterns: An empirical study. Proceedings of the 4th Metaheuristics Int. Conf., University of Porto, Porto, Portugal (pp. 417-421).

Waescher, G., Haussner, H., \& Schumann, H. (2007). An improved typology of cutting and packing problems. Eur J Oper Res, 183, 1109-1130.

Zhang, D., Kang,Y., \& Deng, A. (2006). A new heuristic recursive algorithm for the strip rectangular packing problem. Computers \& Operations Research, 33, 2209-2217. 